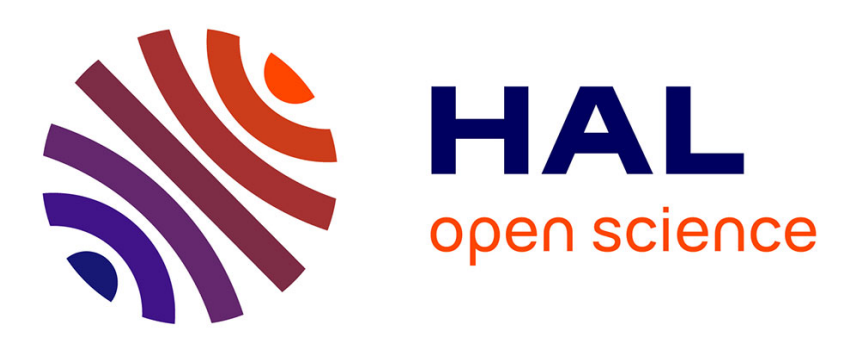

\title{
Drag reduction on drop during impact on multiscale superhydrophobic surfaces
}

Grégoire Martouzet, Choongyeop Lee, Christophe Pirat, Christophe Ybert, Anne-Laure Biance

\section{- To cite this version:}

Grégoire Martouzet, Choongyeop Lee, Christophe Pirat, Christophe Ybert, Anne-Laure Biance. Drag reduction on drop during impact on multiscale superhydrophobic surfaces. Journal of Fluid Mechanics, 2020, 892, 10.1017/jfm.2020.236 . hal-02550195

\section{HAL Id: hal-02550195 \\ https://hal.science/hal-02550195}

Submitted on 5 Nov 2020

HAL is a multi-disciplinary open access archive for the deposit and dissemination of scientific research documents, whether they are published or not. The documents may come from teaching and research institutions in France or abroad, or from public or private research centers.
L'archive ouverte pluridisciplinaire HAL, est destinée au dépôt et à la diffusion de documents scientifiques de niveau recherche, publiés ou non, émanant des établissements d'enseignement et de recherche français ou étrangers, des laboratoires publics ou privés. 


\title{
Drag reduction on drop during impact on multiscale superhydrophobic surfaces
}

\author{
Grégoire Martouzet ${ }^{1}$, Choongyeop Lee ${ }^{2}$, Christophe Pirat $^{1}$, \\ Christophe Ybert $^{1}$ and Anne-Laure Biance ${ }^{1} \dagger$ \\ ${ }^{1}$ ILM, Université de Lyon, Université de Lyon 1 and CNRS, UMR5306, F-69622 Villeurbanne, \\ France \\ ${ }^{2}$ Department of Mechanical Engineering, Kyung Hee University, Yongin 17104, Korea
}

(Received $\mathrm{xx}$; revised $\mathrm{xx}$; accepted $\mathrm{xx}$ )

Liquid drop impact dynamics depends on the liquid-substrate interaction. In particular, when liquid-solid friction is decreased, the spreading of the impacting drop lasts longer. We characterise this effect by using two types of superhydrophobic surfaces, with similar wetting properties but different friction coefficients. It is found that, for large enough impact velocities, a reduced friction delays the build-up of a viscous boundary layer, and leads to an increase of the time required to reach the maximal radius of the impacting drop. An asymptotic analysis is carried out to quantify this effect, and agrees well with the experimental findings. Interestingly, this novel description complements the general picture of drop impact on solid surfaces, and more generally addresses the issue of drag reduction in the presence of slippage for non-stationary flows.

Key words:

\section{Introduction}

Despite being microscopic in nature, the liquid-solid interface can have a strong influence on various macro scale dynamics such as for liquid drop impact on solid surfaces (Yarin 2006), solid body impact into a liquid pool (Duez et al. 2007), and inertial separation between liquid flow and solid (Duez et al. 2010). Drop impact on a solid surface has been of particular interest for understanding the complex physical phenomena involved, as well as for its consequences in many industrial processes (Josserand \& Thoroddsen 2016). Despite its huge interest, the effect of surface wettability on droplet impact dynamics remains debated. On the one hand, experiments show its pronounced role on spreading (Bayer \& Megaridis 2006; Vadillo et al. 2009; Liu et al. 2014) or bouncing (Richard \& Quere 2000) at low impact velocity, whereas others show no influence at larger impact velocities (Clanet et al. 2004; Tsai et al. 2011). Local characterisation even points the crucial effect of trapped air film during impact (de Ruiter et al. 2015). On the other hand, a large number of theoretical, numerical and experimental studies have evidenced that surface friction can play an important role during drop impact at large impact velocity, mainly by considering the two limiting cases of frictionless impact (Clanet et al. 2004; Tsai et al. 2009; Lastakowski et al. 2014), and fully frictional impact with a zero velocity condition at the solid substrate (Roisman et al. 2009; Roisman 2009; Eggers et al. 2010; Schroll et al. 2010; Lagubeau et al. 2012). In the latter case, drop

$\dagger$ Email address for correspondence: anne-laure.biance@univ-lyon1.fr 

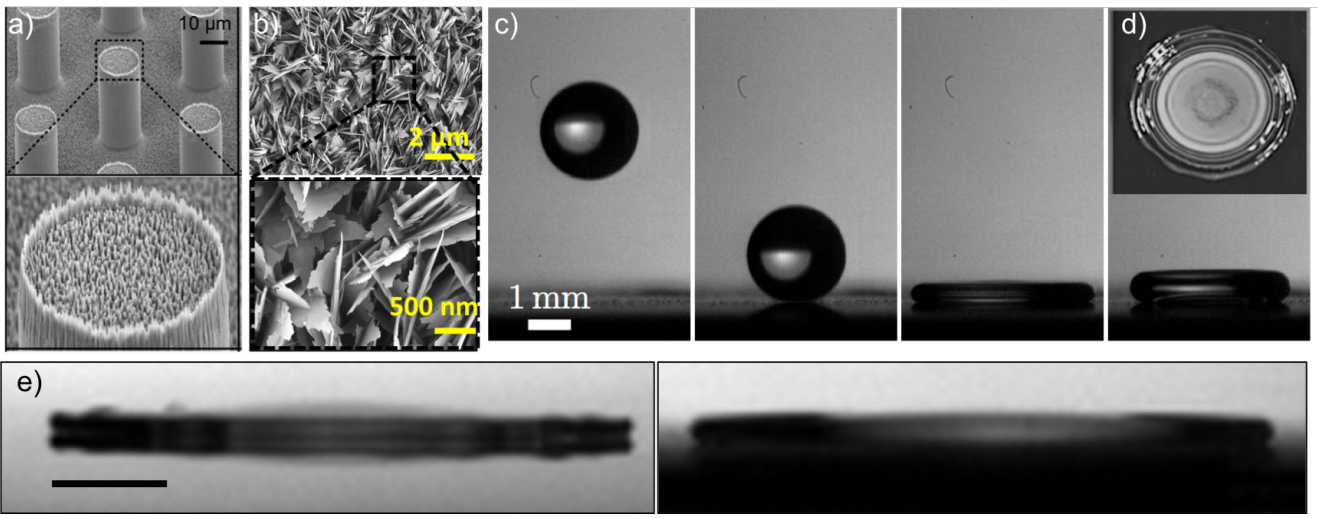

FiguRE 1. Scanning electron microscopy images of : (a) the textured SHPo surfaces B, (b) the disordered SHPo surface C. (c) Snapshot of a $80 \%$ mixture of water and glycerol drop impact on surface B $(U=1.5 \mathrm{~m} / \mathrm{s}$, time interval $2 \mathrm{~ms})$. (d) Top view of a water droplet impact on surface $\mathrm{B}(U=1.5 \mathrm{~m} / \mathrm{s})$. e) Snapshots of the impact at maximal spreading for impact on surface A (left) and B (right). Scale bar corresponds to $1 \mathrm{~mm}$. $(U=2.6 \mathrm{~m} / \mathrm{s}, \eta=21 \mathrm{cS})$.

spreading after impact is governed by the build-up of a viscous boundary layer in the vicinity of the substrate. The case of partial friction has however hardly been explored.

With the development of micro and nanofabrication techniques, it is possible to engineer surfaces with well controlled topography. Particularly, hydrophobic surfaces with micro or nanoscale features (so-called superhydrophobic (SHPo) surfaces) are found to promote unique liquid dynamic characteristics due to the reduced liquid-solid contact (Callies \& Quéré 2005). For example, previously unseen phenomena such as crystallographic splashing (Reyssat et al. 2010; Tsai et al. 2011), pancake bouncing (Liu et al. 2014) and reduced contact time (Bird et al. 2013) were observed upon impact on such surfaces. On SHPo surfaces, the presence of a stress-free liquid-gas interface affects not only the wetting properties of the droplet but also results in a frictional drag reduction, which is commonly quantified using the effective slip length denoted $b$, defined as the ratio of slip velocity to the shear rate at the wall (Joseph et al. 2006; Choi \& Kim 2006; Rothstein 2010; Ybert et al. 2007). This effect has been ignored for impacts of simple fluid drops, as it has been observed to have no consequences on droplet spreading (Clanet et al. 2004; Tsai et al. 2011), although a giant drag reduction has been observed with complex fluids (Luu \& Forterre 2013). Determining how the boundary layer - and the overall impact dynamics - is affected by an intermediate friction is the goal of this study. We focus on a regime at high impact velocities, where the effect of friction on the substrate, quantified by the so-called slip length, is relevant for drop spreading dynamics.

One of the main concerns in conducting quantitative experimental studies on SHPo surfaces is that it can sustain a low frictional Cassie-Baxter state as long as the drop interacts with the top region of surface structures without intruding into them (Lee et al. 2008). However, during drop impact, the dynamic pressure in the liquid drop might overcome the resistance upon imbibition provided by surface tension, leading to the so-called Cassie-to-Wenzel transition (Bartolo et al. 2006; Reyssat et al. 2006). It was proposed that the impalement during impact might be avoided by using SHPo surface with nanometric features (Deng et al. 2009; Tsai et al. 2009), but it would compromise the slip length, as the slip length is proportional to the structural pitch of surface features. This problem can be addressed by employing multi-scale superhydrophobic 


$\begin{array}{lccc}\text { Name } & \text { Type } & \text { Contact angle } & \text { slip length } b \\ \text { A } & \text { Smooth } & \leqslant 30^{\circ} & 0 \mu \mathrm{m} \\ \mathrm{B} & \text { Multiscale ordered } & \geqslant 160^{\circ} & 35 \pm 5 \mu \mathrm{\mu m} \text { (Lee \& Kim 2011) } \\ \text { C } & \text { Multiscale disordered } & \geqslant 160^{\circ} & 8 \pm 1 \mu \mathrm{m}\end{array}$

TABLE 1. The three types of surface used and their characteristics.

surfaces (Lee \& Kim 2011), where microscale features ensure a large slip length, while nanoscale features prevent the detrimental Cassie-to-Wenzel transition.

\section{Experiments}

We designed two types of multiscale superhydrophobic surfaces (Fig. 1a-b, Appendix A), noted as B and C, resulting in both cases in similar large static contact angles, but in different friction characteristics and so different slip lengths for water (Table 1). A bare smooth silicon wafer was used as a reference (surface A).

Drop impact is captured from the side with a high speed camera (Photron SA-4) at up to $30,000 \mathrm{fps}$ (Fig. 1c). The liquids used are mixtures of ultra pure water and glycerol, with a kinematic viscosity $\nu$ ranging from $1 \mathrm{cS}$ (pure water) to $630 \mathrm{cS}$ (pure glycerol). Drops of radius $R \approx 1 \mathrm{~mm}$ are used, with an impact velocity $0.1<U<3.5 \mathrm{~m} / \mathrm{s}$. We introduce as relevant non-dimensional numbers the Weber number We $=\rho R U^{2} / \gamma$, that gives the relative importance of inertia compared to surface tension, and the Reynolds number defined here as $\operatorname{Re}=U R / \nu$. Accordingly, We varies between 40 and 1000, and Re between 8 and 11000. $\tau$, the time to reach the maximum spreading radius, $(t=0$ when the drop reaches the substrate), is a relevant parameter to probe the dynamics. $U$, $R$ and $\tau$ were detected automatically via image analysis using python (Appendix B). For each set of parameters, three experiments are recorded and $\tau$ is averaged.

To confirm that multiscale surfaces ensure robustness towards the Cassie-to-Wenzel transition, a top view image of impact on SPHo surface B is reported in Fig. 1d). One can observe that the region where the transition might have occurred, characterised by small dark dots on the picture, is very limited. More interestingly, dynamical wetting properties seem to be very similar in the three cases considered. In Fig. 1c) and 1e) left an right, corresponding to spreading on surfaces $\mathrm{C}, \mathrm{A}$, and $\mathrm{B}$ respectively, a dynamic contact angle can be measured and is around $140^{\circ} \pm 10^{\circ}$ in both cases, so fully in an hydrophobic regime. We can notice that in our range of impact parameters, lamella lift-off is never observed.

\section{Analysis: scaling laws}

The different regimes of drop impact are summarised in Fig. 2 in the Re-We phase diagram. In the frictionless case, two regimes can be identified, depending on We values. At low We, the inertial timescale to reach the pressure-gradient-free regime $\left(\tau_{i} \sim R / U\right)$ is larger than the timescale of capillary recoil $\left(\tau_{c} \sim \sqrt{\rho R^{3} / \gamma}\right)$ and a pancake regime is observed as described in Clanet et al. (2004), defined by the orange zone of Fig. 2.. At larger We, the rim dynamic limits the drop spreading and $\tau \sim \tau_{c}=\tau_{i} \mathrm{We}^{1 / 2}$ (Eggers et al. 2010; Lastakowski et al. 2014), whatever the impact velocity (Biance et al. 2011). This regime is defined by the yellow region in Fig. 2. We limit our study here to We $>40$, avoiding pancake regimes and coupling between intrusion timescale and spreading (Liu et al. 2014). 
When friction comes into play, a viscous boundary layer builds up from the substrate. It has been demonstrated numerically (Josserand et al. 2005; Eggers et al. 2010) and experimentally (Roisman et al. 2009; Lagubeau et al. 2012; Lastakowski et al. 2014) that the spreading is stopped when the viscous boundary layer meets the upper interface of the thinning lamella. Since pressure-free drop thinning follows $h_{t} \sim R^{3} / U^{2} t^{2}$ (Lastakowski et al. 2014), whereas viscous boundary layer development reads $h_{B L} \sim \sqrt{\nu t}$, the spreading dynamics is stopped for a critical lamella thickness satisfying

$$
\frac{R^{3}}{U^{2} \tau_{v}^{2}} \simeq \sqrt{\nu \tau_{v}},
$$

thus at a viscous time $\tau_{v} \sim \tau_{i} \operatorname{Re}^{1 / 5}$. From the comparison of $\tau_{v}$ with $\tau_{c}$, the viscous regime is dominant over the capillary regime if $\mathrm{We} \gtrsim \mathrm{Re}^{2 / 5}$ (Lagubeau et al. 2012). This corresponds to the grey region of Fig. 2.

We now consider the case of a slippery surface. Introducing the slip length $b$, the no-slip boundary condition is changed for $b u_{z}=\left.u\right|_{z=0}$, where $u$ is the fluid velocity tangential to the surface and the subscript $(z)$ denotes partial differentiation with respect to $z$. From the unsteady 1D momentum diffusion equation $\partial_{t} u=\nu \partial_{z z} u$, it can be shown that a self similar solution for $u$ can be approximated by $u(z, t, b) \simeq U_{f} \frac{z+b}{\sqrt{\pi \nu t}}$ in the vicinity of the surface $(z \sim b)$, where $U_{f}$ is the fluid velocity far from it (Appendix C). Asymptotically, the effect of slippage is to shift by $\sim b$ the viscous boundary layer toward the surface, and $\tau_{s}$, the value of $\tau$ in the slippery case, is thus given by

$$
\frac{R^{3}}{U^{2} \tau_{s}^{2}} \simeq \sqrt{\nu \tau_{s}}-b .
$$

Assuming that the slippage has a weak influence on the dynamics, a perturbation of the viscous case readily leads to

$$
\tau_{s} \simeq \tau_{v}(1+2 \alpha), \quad \text { with } \quad \alpha=\frac{b}{5 \sqrt{\nu \tau_{v}}}=\frac{b}{5 R} \operatorname{Re}^{2 / 5} .
$$

Comparing $\tau_{s}$ with $\tau_{c}$, a new critical transition line can be defined in the Re-We phase space when slippage effects appear. It results that the critical domain for which the capillary-driven regime is observed is extended in the viscous regime, and is given by We $\sim \operatorname{Re}^{2 / 5}(1+4 \alpha)$. This is shown in Fig. 2 where the classical boundary (no-slip) between capillary and viscous regimes is presented by a dotted line, while the new boundary in presence of a slip length $b$ appears as a solid line. We focus then on a new regime that should be caused by a slip-delayed viscous boundary layer development.

\section{Discussion}

We now discuss our experimental results concerning the spreading time reported in Fig. 3. Deviations between the two cases (impact on smooth and SPHo substrates) are observed whatever the Reynolds number. When the spreading time is larger than the capillary time $\tau_{c}$, indicated in Fig. 3 by an orange band, spreading on SPHo surface is shorter than on smooth substrate, a surprising behaviour but which is not in our region of interest. On the contrary, when the spreading time is shorter than the capillary time $\tau_{c}$, the spreading time observed for impact on smooth surfaces A is always shorter than the one on slippery SHPo surfaces B. Even though significant, this increase could be attributed to rim shape or contact angle differences in both cases. To disentangle the effects of wetting and slippage, we performed similar experiments on surface C, which remains superhydrophobic but with a larger friction coefficient. 


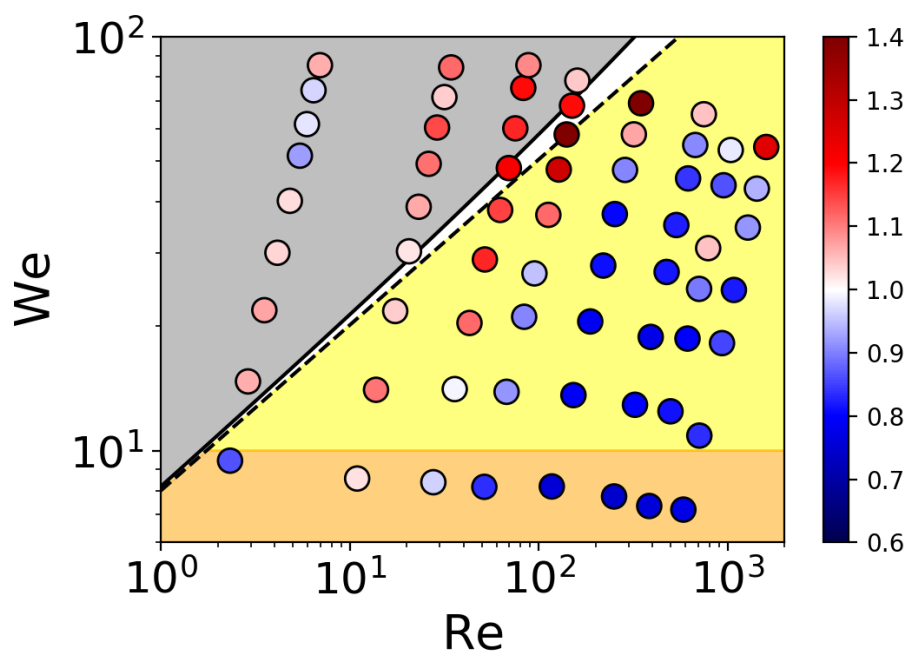

FiguRE 2. Schematic of the drop impact regimes in the We-Re phase diagram. Grey, yellow and orange areas correspond respectively to the viscous, capillary and inertial regimes (see text). In case of total friction, the limit between the capillary regime and viscous regime is determined by the dashed line $\left(\mathrm{We}=8 \mathrm{Re}^{2 / 5}\right)$. In case of partial friction, the capillary regime is extended toward the grey region until the plain line following $\mathrm{We}=8 \mathrm{Re}^{2 / 5}\left(1+0.024 \mathrm{Re}^{2 / 5}\right)$, corresponding to $b=59 \mu \mathrm{m}$. Data points correspond to experiments performed on smooth (A) and rough superhydrophobic surface (B). Color scales correspond to the value of $\tau$ (SPHo) $/ \tau$ (Smooth).

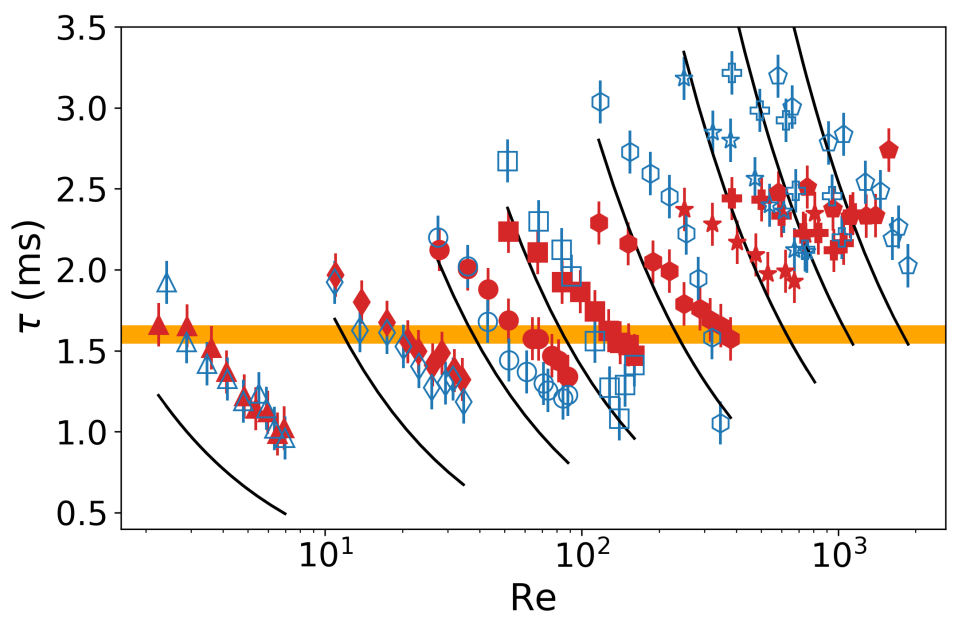

Figure 3. Time $\tau$ to reach the maximal radius versus the Reynolds number on smooth (A, open symbols) and on SHPo (B, plain symbols) substrates with $\nu=505 \mathrm{cS}$ (triangle), $\nu=103 \mathrm{cS}$ (diamond), $\nu=40.5 \mathrm{cS}$ (circle), $\nu=21 \mathrm{cS}$ (square), $\nu=9.4 \mathrm{cS}$ (hexagon), $\nu=4.3 \mathrm{cS}$ (star), $\nu=2.8$ $\mathrm{cS}$ (cross) and $\nu=1.9 \mathrm{cS}$ (pentagon). Plain lines correspond to $\tau=(R / U) \operatorname{Re}^{1 / 5}$ and horizontal band corresponds to the interval containing $\tau=0.41 \sqrt{\rho R^{3} / \gamma}$.

We then report in Fig. 4 the ratio of the spreading time on SHPo surfaces (B or C) on smooth substrate $(\mathrm{A})\left(\tau_{s} / \tau_{v}\right)$ in identical impact conditions as a function of the Reynolds number. Comparing surfaces only differing in their slippage properties allows to evidence a small yet clear experimental effect with surface B showing a delayed spreading that 


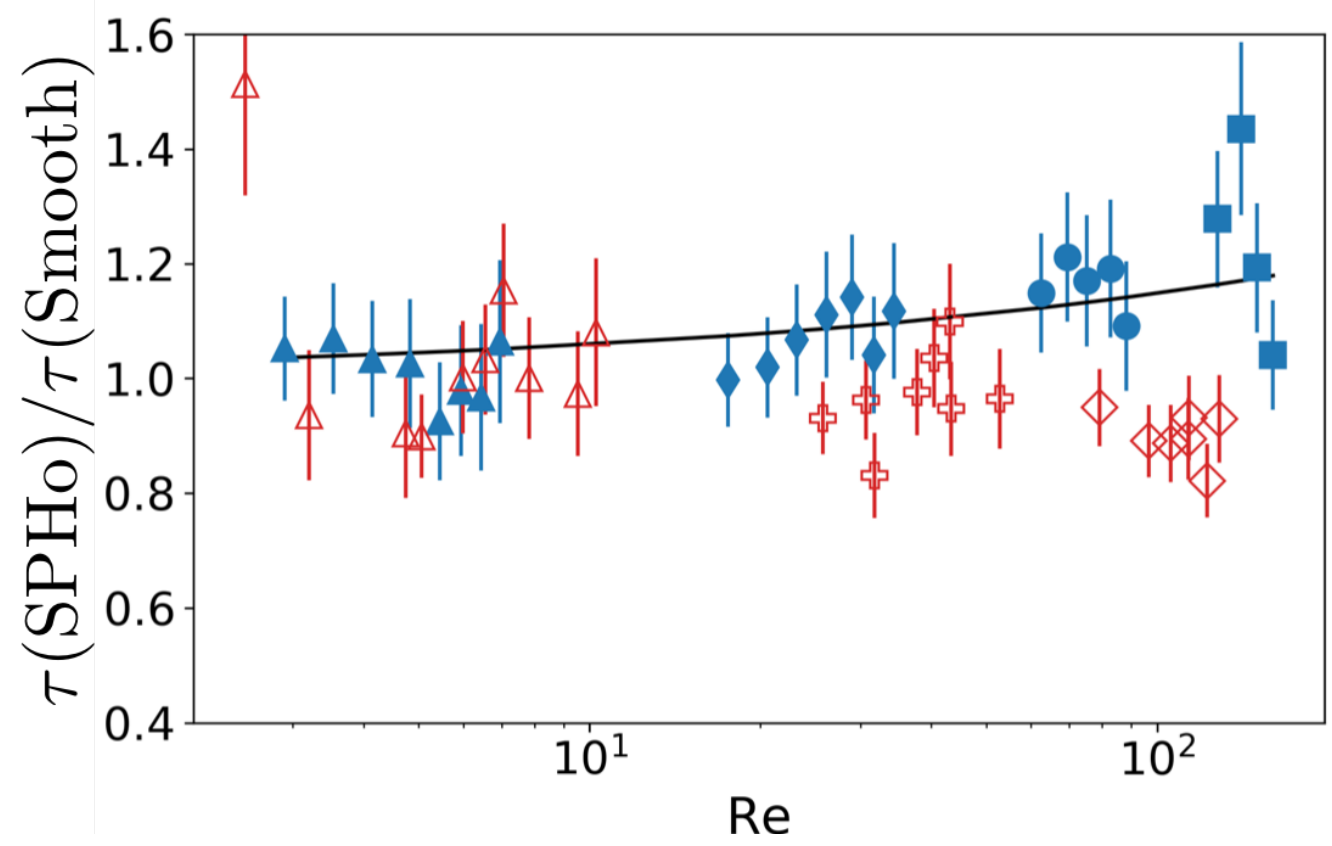

FiguRE 4 . Time $\tau(\mathrm{SHPo})$ to reach the maximal radius on slippery surfaces normalised by the time $\tau$ (Smooth) to reach the maximal radius on smooth surface. Blue plain symbols: ordered rough surface B; Open red symbols: disordered rough surface C. For ordered rough surfaces, viscosities are $\nu=505 \mathrm{cS}$ (triangle), $\nu=103 \mathrm{cS}$ (diamond), $\nu=40.5 \mathrm{cS}$ (circle) and $\nu=21 \mathrm{cS}$ (square). For disorder rough surface, viscosity used are $\nu \geqslant 250 \mathrm{cS}$ (triangle), $\nu=67 \mathrm{cS}$ (cross) and $\nu=27 \mathrm{cS}$ (star). Plain line corresponds to adjustment by eq.3.3 with $b=59 \mu \mathrm{m}$.

increases with Reynolds number. On the contrary surface C shows a spreading essentially identical to a classical no-slip wetting surface, with even a slightly shortened spreading, a behavior we can attribute to either contact line effects (Laan et al. 2014) or fragmentation (Wachters \& Westerling 1966). To show a quantitative agreement, a fit of our data with eq.3.3 is performed allowing us to define an average slip length $b$ of the order of $59 \pm 8 \mu \mathrm{m}$. This value is fully consistent with the slip length obtained by Torque measurements (30$40 \mu \mathrm{m}$ ) for water on the same surfaces (Lee \& Kim 2011). However, considering that our analysis is performed for a $2 \mathrm{D}$ stationary flow, that the slip length varies with the liquid viscosity and nature (here water-glycerol mixtures instead of water), the applied pressure (Lee \& Kim 2011) (here dynamical pressure during impact), as well as a partial transient impalement on the microstructure, the quantitative comparison should be taken cautiously.

To define more generally the range of parameters for which these effects are relevant, we report experimental observations on the Re-We diagram defined above in Fig. 2. The colour bar corresponds to the experimental values of the ratio $\tau(\mathrm{SPHo}) / \tau(\mathrm{Smooth})$. One can indeed observe an increase of this ratio in the viscous regime. A new frontier between the capillary and slippery regimes can be defined by balancing $\tau_{s}$ and $\tau_{c}$ (Fig. 2).

\section{Conclusion}

We propose here a semi-analytical analysis that accounts for drag reduction on viscous impact. A phase diagram is built to better identify the physical parameters for which these effects on the overall dynamics are relevant. It shows the consequences of a weak 
friction on the spreading dynamics, which can be crucial in many situations. To test it, impacts are performed on two types of multiscale SHPo surfaces, that both resist the Cassie-Baxter-to-Wenzel transition and that have similar wetting properties but different friction characteristics. Longer spreading is indeed observed for the lower friction.

Taking into account this effect would be particularly relevant for the design of efficient cooling or anti-icing systems built upon such substrates, where increasing heat transfer through extended contact time and on an extended surface is of crucial interest. On a more fundamental prospect, we propose here an experimental demonstration and a simple analysis of the effect of slippage on the build-up of a viscous boundary layer in a non-stationary flow, a complex mathematical problem (Matthews \& Hill 2008; Gie \& Kelliher 2012), still investigated recently (Fujioka \& Wei 2018).

We thank H. Lastakowski for preliminary experiments. G. Martouzet acknowledges ENS Paris-Saclay for funding, and this work is supported by the Basic Research Program (2017R1A2B4008028) through the National Research Foundation of Korea funded by the Ministry of Science and ICT. This work was sponsored by the French National Agency for Research (Freeflow project ref. ANR-11-B504-001-01). The authors report no conflict of interest.

\section{Appendix A. Surface fabrication and characterization}

Surface B is a well structured surface with multiscale pillars. First, micro-sized cylindrical pillars were obtained using photo-lithography, followed by deep reactive ion etching on silicon. Pillars are arranged in a square lattice with a fixed structural pitch $L$ (centre-to-centre distance of two adjacent pillars) of $50 \mu \mathrm{m}$. The top pillar area fractions $\phi_{s}=\pi D^{2} /\left(4 L^{2}\right)$, with $D$ the pillar diameter, is set to 0.1 . Then, over micro-scale pillars, nano-scale pillars with a structural pitch of $0.5-1 \mu \mathrm{m}$ and very sharp tips were generated using a self-masking nanofabrication method known as a black silicon method (Sun et al. 2010; Lee \& Kim 2011). The surface is made hydrophobic with a few nanometer thick Teflon layer coating. Using torque measurements, the slip length of water on this surface is found to be around $35 \pm 5 \mu \mathrm{m}$.

Surface $\mathrm{C}$ is a randomly textured surface made of $\mathrm{CuO}$ obtained by oxidation of a copper sample in a mixture of $\mathrm{NaClO}_{2}, \mathrm{NaOH}$ and $\mathrm{Na}_{3} \mathrm{PO}_{4}$. It is made hydrophobic by silane vapour deposition. Images of the surface are reported in Fig.1. Slip length from water Poiseuille flow rate measurements in a microfluidic channel is found to be $8 \pm 1$ $\mu \mathrm{m}$. Note that even if slip length measurement methods are different for the two surfaces, they both involve a relatively low liquid pressure and a laminar flow condition, they can be reasonably compared Lee et al. (2016).

Static angles on both surfaces were found to be similar and above $160^{\circ}$. Dynamical contact angle during the spreading are also similar in the three cases of smooth substrate (A) and superhydrophobic surfaces $(\mathrm{B}, \mathrm{C})$, larger than $160^{\circ}$.

\section{Appendix B. Spreading time detection and accuracy}

Typical snapshots of impact recording are reported in Fig.1. The droplet contour and position is determined by substracting the background and thresholding (Fig.5). Results are hardly dependent on the choice of the threshold (1 pixel). The contact time origin is automatically determined by looking at the presence of a bright pixel below the drop. The droplet maximum radius is determined by fitting the evolution of the droplet radius versus time and fitting the curve in the vicinity of the maximum by a second order polynomial. The maximum of the function and the time to reach this maximum correspond then for 


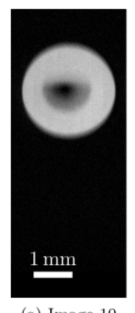

(a) Image 10

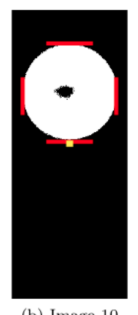

(b) Image 10

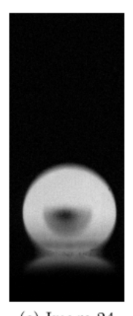

(c) Image 24

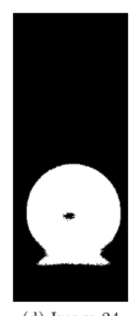

(d) Image 24

Figure 5. Analysed images from a video of a spreading droplet of a $70 \%$ glycerol/water mixture on C. (a) and (c), images with substracted background; (b) and (d), binary images.

each experiment at the spreading radius and spreading time respectively. Finally, the spreading time uncertainty is $0.17 \mathrm{~ms}$.

\section{Appendix C. Boundary layer development over a surface with a Navier condition}

\section{C.1. 2D analysis-Infinite surface}

We consider the motion of a liquid near a solid infinite plane located at $z=0$. The liquid and the plate are initially at rest and then the plate is moved at a velocity $U_{f}$, in the $x$ direction. We investigate the development of a viscous boundary layer in the $z$ direction taking into account this effect. The momentum diffusion equation locally reads:

$$
\frac{\partial u_{x}}{\partial t}=\nu \frac{\partial^{2} u_{x}}{\partial z^{2}}
$$

where $\nu$ is the kinematic viscosity of the liquid.

Introducing $\xi=z / \sqrt{\nu t}$ and the two constants $A$ and $B$, the solution of eq.C 1 is:

$$
u_{x}(z, t)=A \int_{0}^{\xi} e^{-y^{2} / 4} d y+B
$$

Boundary conditions are that (i) the fluid is at rest far from the surface $\left(\lim u_{x}(x \rightarrow\right.$ $+\infty)=0$ ), (ii) in the vicinity of the surface $(z=0)$, a slip condition defined by a slip length $b,\left(\left.b \frac{\partial u_{x}}{\partial z}\right|_{z=0}=\left.u_{x}\right|_{z=0}-U_{f}\right)$. Then, the velocity profile reads:

$$
u_{x}(z, t)=\frac{U_{f}}{\frac{b}{\sqrt{\pi \nu t}}+1}\left(1-\operatorname{erf}\left(\frac{z}{\sqrt{4 \nu t}}\right)\right)
$$

where we recover its usual form for $b=0$. The deviation due to slippage is then

$$
\frac{u_{x}(z, t, b)}{u_{x}(z, t, 0)}=\frac{1}{\frac{b}{\sqrt{\pi \nu t}}+1} .
$$

This term is negligible when $t \geqslant b^{2} / \nu$. As the boundary layer develops from the substrate, it reaches the altitude $z$ after a characteristic time $z^{2} / \nu$, the effect of slippage will be important if $b \geqslant z$. We define the effect of slip on the altitude at which viscous friction is felt versus time. Considering that the viscous friction is felt when $u_{x}(z, t) / U_{f}=k$, with $k$ comprised between 0 and 1 , the time-dependent $z$ at which viscous effects are important reads

$$
z(b, t, k)=\sqrt{4 \nu t} \operatorname{erf}^{-1}\left(1-k-\frac{b k}{\sqrt{\pi \nu t}}\right) .
$$


The effect of slippage can be captured by estimating $z(b, t, k \rightarrow 1)-z(0, t, k \rightarrow 1)$. When $t \rightarrow \infty$, this value is converging to $b$. The boundary layer is shifted up by a length $b$.

\section{C.2. Consequences on droplet dynamics upon impact: timescales}

We consider a drop impacting on a solid substrate.We distinguish here the three cases of drop impact without friction (i.e. Leidenfrost case), with large friction (smooth solid substrate), and with slippage (superhydrophobic substrate). For each regime, we identify the characteristic time to reach the maximal radius and these times will be compared to identify which regime is predominant as a function of the Weber $\mathrm{We}=\frac{\rho R^{3}}{\gamma}$ and Reynolds $\operatorname{Re}=\frac{U R}{\nu}$ numbers.

\section{C.2.1. Frictionless case}

In this regime, two cases can be determined depending only on the Weber number. A deceleration phase over a time $\tau_{i} \sim R / U$ (Clanet et al. 2004) is followed by a capillary oscillation over $\tau_{c} \sim \sqrt{\frac{\rho R^{3}}{\gamma}}$. If $\tau_{c}$ is smaller than $\tau_{i}$, only the deceleration is observed and we reach a pancake regime whereas on the contrary a capillary recoil (Roisman et al. 2009; Eggers et al. 2010; Lastakowski et al. 2014) is observed. The transition is given by $\tau_{c}=\tau_{i}$, which implies a critical Weber number $\mathrm{We}_{1}$. In the following analysis, we assume that We is always above $\mathrm{We}_{1}$.

\section{C.2.2. Large friction}

In this case, it has been shown that the development of a boundary layer sets the maximal attained radius, i.e. when the droplet thickness is comparable to this developed boundary layer, the droplet stops spreading and thinning. For We larger than $\mathrm{We}_{1}$, this criterion reads (Roisman et al. 2009; Eggers et al. 2010; Lastakowski et al. 2014)

$$
\frac{R^{3}}{U^{2}\left(\tau_{v}\right)^{2}} \simeq k \sqrt{\nu\left(\tau_{v}\right)}
$$

and then, the spreading viscous time scales as $\tau_{v} \sim \tau_{i} R e^{1 / 5}$. This regime is relevant if this time is shorter than $\tau_{c}$, i.e. We $\geqslant \operatorname{Re}^{2 / 5}$.

\section{C.2.3. Slippage}

Following previous asymptotic analysis, the characteristic time $\tau_{s}$ to develop the boundary layer over the distance $h$ satisfies:

$$
\frac{R^{3}}{U^{2} \tau_{s}^{2}} \simeq k \sqrt{\nu \tau_{s}}-b .
$$

Let's now assume that the effect of slippage is small compared to viscous effects. The characteristic viscous slippery time $\tau_{s}$ can then be written

$$
\tau_{s}=\tau_{v}+t_{s}
$$

with $t_{s}=o\left(\tau_{v}\right)$ is the slippage contribution. Eq. (C 7) can then be developed as

$$
\begin{gathered}
-\frac{R^{3}}{U^{2} \tau_{v}^{2}} \frac{2 t_{s}}{\tau_{v}} \simeq k \sqrt{\nu \tau_{v}} \frac{t_{s}}{2 \tau_{v}}-b \\
\tau_{s} \simeq \tau_{v}\left(1+\frac{2 b}{5 R} \operatorname{Re}^{2 / 5}\right) .
\end{gathered}
$$


The capillary region is then extended to $\mathrm{We} \lesssim \operatorname{Re}^{2 / 5}\left(1+\frac{2 b}{5 R} \operatorname{Re}^{2 / 5}\right)$ (Fig.2).

\section{REFERENCES}

Bartolo, D., Eouamrirene, F., Verneull, E., Buguin, A., Silberzan, P. \& Moulinet, S. 2006 Bouncing or sticky droplets: Impalement transitions on superhydrophobic micropatterned surfaces. Europhys. Lett. 74, 299-305.

Bayer, I. S. \& Megaridis, C. M. 2006 Contact angle dynamics in droplets impacting on flat surfaces with different wetting characteristics. Journal of Fluid Mechanics 558, 415-449.

Biance, A.-L., Pirat, C. \& Ybert, C. 2011 Drop fragmentation due to hole formation during Leidenfrost impact. Phys Fluids 23 (2), 022104.

Bird, J. C., Dhiman, R., Kwon, H.-M. \& Varanasi, K.K. 2013 Reducing the contact time of a bouncing drop. Nature $\mathbf{5 0 3}$ (7476), 385-388.

Callies, M. \& Quéré, D. 2005 On water repellency. Soft matter 1 (1), 55-61.

Chor, C.-H. \& KIm, C.-J. 2006 Large slip of aqueous liquid flow over a nanoengineered superhydrophobic surface. Physical review letters 96 (6), 066001.

Clanet, C., Beguin, C., Richard, D. \& Quere, D. 2004 Maximal deformation of an impacting drop. J. Fluid Mech. 517, 199.

Deng, T., Varanasi, K. K., Hsu, M., Bhate, N., Keimel, C., Stein, J. \& Blohm, M. 2009 Nonwetting of impinging droplets on textured surfaces. Appl. Phys. Lett. 94, 133109.

Duez, C., Ybert, C., Clanet, C. \& Bocquet, L. 2007 Making a splash with water repellency. Nature Physics 3 (3), 180-183.

Duez, C., Ybert, C., Clanet, C. \& Bocquet, L. 2010 Wetting controls separation of inertial flows from solid surfaces. Physical review letters 104 (8), 084503.

Eggers, J., Fontelos, M. A., Josserand, C. \& Zaleski, S. 2010 Drop dynamics after impact on a solid wall: Theory and simulations. Phys. Fluids 22, 062101.

FujiokA, H. \& WeI, H.-H. 2018 Letter: New boundary layer structures due to strong wall slippage. Physics of Fluids 30 (12), 121702.

Gie, Gung-Min \& Kelliher, James P. 2012 Boundary layer analysis of the navier-stokes equations with generalized navier boundary conditions. Journal of Differential Equations 253 (6), 1862-1892.

Joseph, P., Cottin-Bizonne, C., Benoit, J.-M., Ybert, C., Journet, C., Tabeling, P. \& BocQueT, L. 2006 Slippage of water past superhydrophobic carbon nanotube forests in microchannels. Physical review letters 97 (15), 156104.

Josserand, C., Lemoyne, L., Troeger, R. \& Zaleski, S. 2005 Droplet impact on a dry surface: triggering the splash with a small obstacle. J. Fluid Mech. 524, 47.

Josserand, C. \& Thoroddsen, S.T. 2016 Drop impact on a solid surface. Annual Review of Fluid Mechanics 48 (1), 365-391.

Laan, Nick, de Bruin, Karla G., Bartolo, Denis, Josserand, Christophe \& Bonn, Daniel 2014 Maximum diameter of impacting liquid droplets. Phys. Rev. Applied 2, 044018 .

Lagubeau, G., Fontelos, M. A., Josserand, C., Maurel, A., Pagneux, V. \& Petitjeans, P. 2012 Spreading dynamics of drop impacts. Journal of Fluid Mechanics 713, 50-60.

Lastakowski, H., Boyer, F., Biance, A.-L., Pirat, C. \& Ybert, C. 2014 Bridging local to global dynamics of drop impact onto solid substrates. J Fluid Mech 747, 103-118.

Lee, C., Chor, C.-H. \& Kim, C.J. 2008 Structured surfaces for a giant liquid slip. Phys. Rev. Lett. 101, 064501.

LEE, C. \& KIM, C.J. 2011 Underwater restoration and retention of gases on superhydrophobic surfaces for drag reduction. Phys. Rev. Lett. 106, 014502.

Lee, JB, Laan, Nick, de Bruin, Karla G, Skantzaris, G, Shahidzadeh, Noushine, Derome, Dominique, Carmeliet, J \& Bonn, Daniel 2016 Universal rescaling of drop impact on smooth and rough surfaces. Journal of Fluid Mechanics $\mathbf{7 8 6}$.

Liu, Y., Moevius, L., Xu, X., Qian, T., Yeomans, J. M. \& Wang, Z. 2014 Pancake bouncing on superhydrophobic surfaces. Nat Phys 10 (7), 515-519.

LuU, L.-H. \& ForTerre, Y. 2013 Giant drag reduction in complex fluid drops on rough hydrophobic surfaces. Physical review letters 110 (18), 184501. 
Matthews, Miccal T. \& Hill, James M. 2008 A note on the boundary layer equations with linear slip boundary condition. Applied Mathematics Letters 21 (8), 810 - 813.

Reyssat, M., Pein, A., Marty, F., Chen, Y. \& Quere, D. 2006 Bouncing transitions on microtextured materials. Europhys. Lett. 74, 306-312.

Reyssat, M., Richard, D., Clanet, C. \& Quere, D. 2010 Dynamical superhydrophobicity. Faraday Discuss. 146, 19.

Richard, D. \& Quere, D. 2000 Bouncing water drops. Europhys. Lett. 50, 769-775.

Roisman, I. V. 2009 Inertia dominated drop collisions. ii. an analytical solution of the navierstokes equations for a spreading viscous film. Physics of Fluids 21 (5), 052104.

Roisman, I. V., Berberovic, E. \& Tropea, C. 2009 Inertia dominated drop collisions. I. On the universal flow in the lamella. Phys. Fluids 21, 052103.

Rothstein, J. P. 2010 Slip on superhydrophobic surfaces. Annual Review of Fluid Mechanics 42, 89-109.

De Ruiter, Jolet, Lagraauw, Rudy, van den Ende, Dirk \& Mugele, Frieder 2015 Wettability-independent bouncing on flat surfaces mediated by thin air films. Nature Physics 11 (1), 48-53.

Schroll, R. D., Josserand, C., Zaleski, S. \& Zhang, W. W. 2010 Impact of a viscous liquid drop. Phys. Rev. Lett. 104, 034504.

Sun, Guangyi, Gao, Tianle, Zhao, Xin \& Zhang, Haixia 2010 Fabrication of micro/nano dual-scale structures by improved deep reactive ion etching. Journal of Micromechanics and Microengineering 20 (7), 075028.

Tsai, P., Hendrix, M.H.W., Dijkstra, R.M., Shui, L. \& Lohse, D. 2011 Microscopic structure influencing macroscopic splash at high weber number. Soft Matter 7 (24), 1132511333.

Tsai, P., Pacheco, S., Pirat, C., Lefferts, L. \& Lohse, D. 2009 Drop Impact upon Microand Nanostructured Superhydrophobic Surfaces. Langmuir 25, 12293.

Vadillo, D.C ., Soucemarianadin, A., Delattre, C. \& Roux, D. C. D. 2009 Dynamic contact angle effects onto the maximum drop impact spreading on solid surfaces. Physics of Fluids (1994-present) 21 (12), 122002.

Wachters, L. H. \& Westerling, N. A. 1966 Heat Transfer From A Hot Wall To Impinging Water Drops In Spheroidal State. Chemical Engineering Science 21, 1047.

YARIN, A. L. 2006 Drop impact dynamics: Splashing, spreading, receding, bouncing... Ann. Rev. Fluid Mech. 38, 159.

Ybert, C., Barentin, C., Cottin-Bizonne, C., Joseph, P. \& Bocquet, L. 2007 Achieving large slip with superhydrophobic surfaces: Scaling laws for generic geometries. Physics of Fluids (1994-present) 19 (12), 123601. 